\title{
A TRACE FORMULA FOR SCHRÖDINGER OPERATORS WITH STEP POTENTIALS
}

\author{
D. M. O'BRIEN'
}

(Received 9 November 1981)

\begin{abstract}
This paper shows how to compute the trace of $G(T)-G\left(T^{0}\right)$, where $G$ is an infinitely differentiable function with compact support, and where $T$ and $T^{0}$ are one-dimensional Schrödinger operators on $(-\infty, \infty)$ with potentials $q$ and $q^{0}$. It is assumed that $q^{0}$ is a simple step potential and that $q$ decays exponentially to $q^{0}$. The trace is expressed in terms of the reflection and transmission coefficients for the scattering of plane waves by the potential $q$.
\end{abstract}

\section{Introduction}

In this paper we will consider a one-dimensional Schrödinger operator

$$
T=-d^{2} / d x^{2}+q(x), \quad-\infty<x<\infty,
$$

obtained by perturbation from the operator

$$
T^{0}=-d^{2} / d x^{2}+q^{0}(x), \quad-\infty<x<\infty,
$$

wth a simple step potential,

$$
q^{0}(x)= \begin{cases}q_{+}, & x \geqslant 0 \\ q_{-}, & x<0\end{cases}
$$

where

$$
-\infty<q_{-} \leqslant q_{+}<\infty
$$

We will require that the potential $q$ be a real, infinitely differentiable function

\footnotetext{
'Department of Mathematical Physics, University of Adelaide, G.P.O. Box 498, Adelaide, South Australia 5001.

(c) Copyright Australian Mathematical Society 1982
} 
which satisfies

$$
\int_{0}^{\infty} x \exp (s x)\left|q( \pm(x-a))-q_{ \pm}\right| d x<\infty
$$

for any $a \geqslant 0$ and for all $s<2 b$, where $b$ is a fixed, positive constant. Our aim is to show that the operator

$$
Q=G(T)-G\left(T^{0}\right)
$$

is nuclear for any infinitely differentiable function $G$ whose support is compact, and to express the trace of $Q$ in terms of the coefficients of reflection $\left(r_{ \pm}\right)$and transmission $\left(t_{ \pm}\right)$for the scattering of plane waves by the potential $q$. We will establish the following result.

THEOREM 1. The symmetric operator defined by the formally self-adjoint differential expression (1.1) is essentially self-adjoint. Its closure, also denoted by $T$, has at most a finite number of eigenvalues $\lambda_{1}, \lambda_{2}, \ldots, \lambda_{n}$ in the interval $\left(-\infty, q_{-}\right]$, and the continuous spectrum of $T$ is the interval $\left[q_{-}, \infty\right)$. The operator $Q$ is nuclear and its trace is given by the following formula.

$$
\begin{aligned}
\operatorname{trace} Q= & \sum_{i=1}^{n} G\left(\lambda_{i}\right)+\frac{1}{2 \pi i} \int_{q_{-}}^{q_{+}} d \lambda G(\lambda) \frac{d}{d \lambda} \log \left(r_{-} / r_{-}^{0}\right) \\
& +\frac{1}{2 \pi i} \int_{q_{+}}^{\infty} d \lambda G(\lambda) \frac{d}{d \lambda} \log \left(t_{-} / \bar{t}_{-}\right)
\end{aligned}
$$

This work was prompted by a problem in quantum field theory involving the renormalisation of a quantum soliton field in two space-time dimensions (Faddeev and Korepin [9], Lohe [11], Lohe and O'Brien [12]). The classical soliton is a stable solution of a non-linear wave equation which has a conserved 'charge' and whose 'mass' is just the energy of the wave. In the quantum picture, the soliton interacts with the surrounding meson cloud and its mass acquires a correction, which is given to first order in perturbation theory by

$$
\Delta m=\operatorname{trace} T^{1 / 2},
$$

where $T$ is the Schrödinger operator whose potential represents the static field produced by the soliton and experienced by the mesons. This formula, obtained by purely formal manipulations, is divergent and must be renormalised. Firstly, the vacuum energy must be subtracted, and this corresponds to writing

$$
\Delta m=\operatorname{trace}\left(T^{1 / 2}-T^{0^{1 / 2}}\right),
$$

where the potential $q^{0}$ is the residual potential far to the left and right of the soliton. This formula still contains a divergence, caused by the high energy end of 
the spectrum of $T$, which can be cancelled by the addition of a renormalisation counter-term to the Lagrangian. Equivalently, we can write

$$
\Delta m=\operatorname{trace}\left(G(T)-G\left(T^{0}\right)\right),
$$

where $G$ is an infinitely differentiable function with compact support which coincides with $\lambda^{1 / 2}$ on $\left[\Lambda^{-1}, \Lambda\right]$, and then modify the Lagrangian by the addition of a finite counter-term. We must then prove that the counter-term cancels the high energy divergence as $\Lambda \rightarrow \infty$.

For the final step, we need asymptotic developments for the reflection and transmission coefficients, since these appear in the trace formula and determine the asymptotic behaviour of the trace for large $\Lambda$. In the final section of the paper we will show how these developments may be obtained to any order. In particular, we will obtain the following simple result.

LEMMA 1. The transmission coefficients $t_{ \pm}$have the asymptotic form

$$
t_{ \pm} \sim 1+\frac{1}{2 i \lambda^{1 / 2}} \int_{-\infty}^{\infty}\left(q-q^{0}\right)(x) d x+O\left(\lambda^{-1}\right) \text { as } \lambda \rightarrow+\infty .
$$

This example of the renormalisation of the soliton mass is not atypical of many problems in quantum field theory, because it contains the following steps:

(1) we encounter a formal expression which is divergent, but which can be made finite by the introduction of 'cut-offs', or convergence factors;

(2) we perform one or more subtractions to eliminate the divergences;

(3) we attempt to remove the cut-offs.

For models in two space-time dimensions these steps are usually tractable, but in higher dimensions the situation is very obscure.

Several Russian authors, notably Buslaev and Faddeev, have studied the renormalisation problem, both abstractly and in particular cases. Buslaev [3] has shown the existence of a trace formula analogous to (1.3) for a wide class of perturbations which leave unchanged the continuous spectrum of $T^{0}$. In his work the operator $T^{0}$ may be arbitrary, save only that its spectrum be purely continuous and its spectral representation be known, and he represents the perturbation by an integral operator on the spectrum of $T^{0}$. His conditions on the perturbation are formulated in terms of its kernel, and are rather hard to check. At a less abstract level, Buslaev and Faddeev [4] renormalised the trace of the resolvent of the one-dimensional Schrödinger operator defined on the half-line $[0, \infty)$, and more recently Faddeev and Korepin [9, Appendix 6] extended the result to potentials defined on $(-\infty, \infty)$ which decay to zero as $x \rightarrow \pm \infty$. Thus, the aim of this paper is a further extension to potentials which approach unequal limits as $x \rightarrow \pm \infty$. 
The idea of studying scattering from potentials for which the regions to the left and right of the target are asymptotically different is not new. Alsholm and Kato [1] proved the existence of modified wave operators for scattering from long range potentials. Ruijsenaars and Bongaarts [14] considered the one-dimensional Dirac equation with potentials which were constant outside a compact region, but which did not necessarily have equal asymptotic values. They proved the existence of modified wave operators and the unitarity of the $S$-matrix. Davies and Simon [5] have given an elegant account of scattering theory for systems with different spatial asymptotics on the left and right. Some of the basic properties of the operator $T$ (such as its self-adjointness and essential spectrum) could have been deduced from the general analysis in the above references, but, since $T$ is only an ordinary differential operator, all the properties we need can either be established directly or extracted from the comprehensive treatise by Dunford and Schwartz [7].

The first step in the proof of the theorem will be to represent $G(T)$ as an integral operator on $L^{2}(\mathbf{R})$ with $\operatorname{kernel} G(x, y)$,

$$
\begin{gathered}
G(T): L^{2}(\mathbf{R}) \rightarrow L^{2}(\mathbf{R}), \\
f_{\mapsto}(G(T) f)(x)=\int_{\mathbf{R}} G(x, y) f(y) d y, \\
\mathbf{R}=(-\infty, \infty) .
\end{gathered}
$$

This is a fairly routine task, because the spectral theory of ordinary differential operators is very well established, and the final expression for $G(x, y)$ could be stated without proof. However, there are several pitfalls for the unwary (noted even by Dunford and Schwartz [7, page 1347]!), so we will outline the construction. For this purpose we will adhere to the notation used by Dunford and Schwartz, and frequently quote theorems from their treatise.

Given now that $G(T)$ and $G\left(T^{0}\right)$ are integral operators on $L^{2}(\mathbf{R})$ with kernels $G(x, y)$ and $G^{0}(x, y)$, then so too is $Q$ with kernel

$$
Q(x, y)=G(x, y)-G^{0}(x, y) .
$$

The second step is to prove that $Q$ is nuclear and that its trace is given by

$$
\text { trace } Q=\int_{-\infty}^{\infty} Q(x, x) d x
$$

Finally, we must show that (1.5) reduces to (1.3). The convergence of the integral in (1.5) is rather delicate and we will need some analytical properties of the reflection and transmission coefficients in order to establish it. These, along with precise definitions of $r_{ \pm}$and $t_{ \pm}$, are presented in Section 3, and again the proofs are only outlined, for they are straightforward once the objective is known. 


\section{Scattering solutions}

The first task is the construction of a full complement of solutions of the Schrödinger equation,

$$
\left(-\frac{d^{2}}{d x^{2}}+q(x)\right) \psi=\lambda \psi
$$

which are asymptotically free for large $|x|$, in the sense that they degenerate into solutions of

$$
\left(-\frac{d^{2}}{d x^{2}}+q^{0}(x)\right) \psi=\lambda \psi
$$

To achieve this, we note the following theorem, which can be traced to Jost [10] and Bargmann [2], although more detailed accounts are given in the text on potential scattering by de Alfaro and Regge [6] and the review by Faddeev [8].

THEOREM 2. If the potential $v$ satisfies the condition,

$$
\int_{0}^{\infty} d x x \exp (s x)|v(x)| d x<\infty \text { for all } s<2 b,
$$

then the integral equation,

$$
\psi(x)=\exp (i k x)-k^{-1} \int_{x}^{\infty} \sin k(x-y) v(y) \psi(y) d y,
$$

has a solution $\psi$ with the following properties.

(1) At all points of the region

$$
x>0, \quad \operatorname{Imag} k>-b,
$$

$\psi(x, k)$ is twice differentiable with respect to $x$ and holomorphic in $k$.

(2) $\psi$ satisfies the differential equation

$$
\left(-\frac{d^{2}}{d x^{2}}+v(x)\right) \psi(x, k)=k^{2} \psi(x, k), \quad 0 \leqslant x<\infty .
$$

(3) For every positive integer $m$,

$$
\lim _{x \rightarrow \infty}\left|x^{m}(\exp (-i k x) \psi(x, k)-1)\right|=0,
$$

so $\psi$ converges to the solution of the free wave equation more rapidly than any power of $x$.

Proof. In order to prove this theorem, we solve the integral equation (2.2) by iteration:

$$
\psi=\sum_{n=0}^{\infty} \psi^{n}
$$


where

$$
\psi^{0}(x, k)=\exp (i k x)
$$

and

$$
\psi^{n+1}(x, k)=-k^{-1} \int_{x}^{\infty} \sin k(x-y) v(y) \psi^{n}(y, k) d y .
$$

To establish the absolute and uniform convergence of the series in the region (2.3), we use the inequality,

$$
\left|k^{-1} \sin k(x-y)\right| \leqslant 2^{1 / 2}|x-y| \exp |\operatorname{Imag} k(x-y)|,
$$

and prove by induction that

$$
\left|\psi^{n}(x, k)\right| \leqslant \exp (-\operatorname{Imag} k x) u(x)^{n} / n !
$$

where

$$
u(x)=2^{1 / 2} \int_{x}^{\infty} d y y|v(y)| \exp [(|\operatorname{Imag} k|-\operatorname{Imag} k) y] .
$$

We want to adapt this theorem to the Schrödinger equation on $\mathbf{R}$ with a step potential, so we set

$$
k_{\alpha}=\left(\lambda-q_{\alpha}\right)^{1 / 2}, \quad \alpha= \pm,
$$

and apply the theorem to the overlapping intervals $[-a, \infty)$ and $(-\infty, a]$. Firstly, we let

$$
v(x)=q(x-a)-q_{+}, \quad x \geqslant 0,
$$

and

$$
k=k_{+}
$$

in the preceding analysis, construct the solution $\psi$ of (2.2), and define

$$
\begin{array}{ll}
\psi_{++}\left(x, k_{+}\right)=\exp \left(-i k_{+} a\right) \psi\left(x+a, k_{+}\right), & x \geqslant-a, \quad+\operatorname{Imag} k_{+}>-b, \\
\psi_{+-}\left(x, k_{+}\right)=\exp \left(+i k_{+} a\right) \overline{\psi\left(x+a, \bar{k}_{+}\right)}, & x \geqslant-a, \quad-\operatorname{Imag} k_{+}>-b .
\end{array}
$$

Secondly, we let

$$
v(x)=q(-x+a)-q_{-}, \quad x \geqslant 0,
$$

and

$$
k=k_{-}
$$

in the preceding analysis, construct the solution $\psi$ of (2.2), and define

$$
\begin{array}{lll}
\psi_{-+}\left(x, k_{-}\right)=\exp \left(+i k_{-} a\right) \overline{\psi\left(-x+a, \bar{k}_{-}\right)}, & x \leqslant+a, & -\operatorname{Imag} k_{-}>-b, \\
\psi_{--}\left(x, k_{-}\right)=\exp \left(-i k_{-} a\right) \psi\left(-x+a, k_{-}\right), & x \leqslant+a, & +\operatorname{Imag} k_{-}>-b .
\end{array}
$$

We obtain the following corollary. 
COROLlaRY. Under the stated assumption on the potential (1.2), the integral equation

$$
\psi_{\alpha \beta}\left(x, k_{\alpha}\right)=\exp \left(i \beta k_{\alpha} x\right)+k_{\alpha}^{-1} \int_{\alpha \infty}^{x} \sin k_{\alpha}(x-y)\left(q-q_{\alpha}\right)(y) \psi_{\alpha \beta}\left(y, k_{\alpha}\right) d y,
$$

in which $\alpha$ and $\beta$ may denote either + or - , has a solution $\psi_{\alpha \beta}$ with the following properties.

(1) At all points of the region

$$
\alpha x>-a, \quad \alpha \beta \operatorname{Imag} k_{\alpha}>-b,
$$

$\psi_{\alpha \beta}\left(x, k_{\alpha}\right)$ is twice differentiable with respect to $x$ and holomorphic in $k_{\alpha}$.

(2) $\psi_{\alpha \beta}$ satisfies the differential equation

$$
\left(-\frac{d^{2}}{d x^{2}}+q(x)\right) \psi_{\alpha \beta}\left(x, k_{\alpha}\right)=\lambda \psi_{\alpha \beta}\left(x, k_{\alpha}\right), \quad-a \leqslant \alpha x<\infty .
$$

(3) For every positive integer $m$,

$$
\lim _{\alpha x \rightarrow \infty}\left|x^{m}\left(\exp \left(-i \beta k_{\alpha} x\right) \psi_{\alpha \beta}\left(x, k_{\alpha}\right)-1\right)\right|=0,
$$

so $\psi_{\alpha \beta}$ converges to the solution of the free wave equation more rapidly than any power of $x$.

The strong assumption (1.2) on the potential, that it should decay exponentially to $q^{0}$, is needed to prove that $\psi_{\alpha \beta}$ will be a holomorphic function of $k_{\alpha}$ in an open region containing the positive real axis, a property that will be important later in the Titchmarsh-Weyl-Kodaira algorithm for the spectral measure of $T$. In fact, the weaker condition

$$
\left|q(x)-q^{0}(x)\right| \leqslant c /(1+|x|)^{s}, \quad s>1,
$$

is sufficient to prove the existence of solutions with the asymptotic forms,

$$
\psi_{\alpha \beta}\left(x, k_{\alpha}\right) \sim \exp \left(i \beta k_{\alpha} x\right) \text { as } \alpha x \rightarrow \infty,
$$

but then $\psi_{\alpha \beta}$ will only be holomorphic in the region

$$
\alpha \beta \operatorname{Imag} k_{\alpha}>0
$$

and continuous on the real axis, except possibly for the point $k_{\alpha}=0$. With the stronger conditions,

$$
\int_{0}^{\infty} x|q( \pm(x-a))-q \pm| d x<\infty,
$$

$\psi_{\alpha \beta}$ will be continuous on the whole real axis, but it still might not be possible to analytically continue $\psi_{\alpha \beta}$ into the region

$$
\alpha \beta \operatorname{Imag} k_{\alpha}<0 \text {. }
$$


Note the important identity,

$$
\overline{\psi_{\alpha+}\left(x, \bar{k}_{\alpha}\right)}=\psi_{\alpha-}\left(x, k_{\alpha}\right),
$$

which follows easily from the integral equation satisfied by $\psi_{\alpha \beta}$.

Now $k_{+}$and $k_{-}$are not independent variables, but are related to $\lambda$ through (2.7). We will assume that the branch cut lies along $\left(-\infty, q_{\alpha}\right]$, and adopt the convention that $k_{\alpha}$ and $\psi_{\alpha \beta}$, now regarded as functions of $\lambda$, are defined on the cut by their boundary values from above. Thus,

$$
\left.\begin{array}{l}
k_{\alpha}(\mu)=\lim _{\nu \rightarrow 0+} k_{\alpha}(\mu+i \nu) \\
\psi_{\alpha \beta}(x, \mu)=\lim _{\nu \rightarrow 0+} \psi_{\alpha \beta}(x, \mu+i \nu)
\end{array}\right\}, \quad \mu<q_{\alpha} .
$$

When $\lambda$ lies on the cut $\psi_{\alpha \beta}$ is real,

$$
\overline{\psi_{\alpha \beta}(x, \lambda)}=\psi_{\alpha \beta}(x, \lambda), \quad \lambda \in\left(-\infty, q_{\alpha}\right]
$$

but for all other values

$$
\overline{\psi_{\alpha+}(x, \bar{\lambda})}=\psi_{\alpha-}(x, \lambda), \quad \lambda \notin\left(-\infty, q_{\alpha}\right],
$$

since

$$
\overline{k_{\alpha}(\bar{\lambda})}=k_{\alpha}(\lambda), \quad \lambda \notin\left(-\infty, q_{\alpha}\right]
$$

\section{Reflection and transmission coefficients}

All four solutions $\psi_{++}, \psi_{+_{-}}$and $\psi_{-+}, \psi_{--}$are defined in the region $|x| \leqslant a$, and consequently must be linearly related,

$$
\begin{aligned}
& \psi_{-+}(x, \lambda)=r_{-}(\lambda) \psi_{--}(x, \lambda)+t_{+}(\lambda) \psi_{++}(x, \lambda), \\
& \psi_{+-}(x, \lambda)=r_{+}(\lambda) \psi_{++}(x, \lambda) t_{-}(\lambda) \psi_{--}(x, \lambda) .
\end{aligned}
$$

The coefficients $r_{ \pm}$and $t_{ \pm}$, defined by these identities, are the reflection and transmission coefficients, so named because in (3.1) $\psi_{-+}$representes a plane wave incident on the potential from the left, $\psi_{--}$is the reflected wave retreating to $-\infty$, and $\psi_{++}$is the transmitted wave advancing to $+\infty$. Equation (3.2) can be interpreted similarly. The reflection and transmission coefficients can be expressed in terms of the Wronskians of the asymptotically free solutions,

$$
\begin{array}{rlrl}
r_{+} & =\frac{W\left(\psi_{+-}, \psi_{--}\right)}{W\left(\psi_{++}, \psi_{--}\right)}, & t_{+} & =\frac{W\left(\psi_{-+}, \psi_{--}\right)}{W\left(\psi_{++}, \psi_{++}\right)}, \\
r_{-} & =\frac{W\left(\psi_{-+}, \psi_{++}\right)}{W\left(\psi_{--}, \psi_{++}\right)}, & t_{-}=\frac{W\left(\psi_{+-}, \psi_{++}\right)}{W\left(\psi_{--}, \psi_{++}\right)},
\end{array}
$$


where

$$
W(f, g)=f^{\prime} g-f g^{\prime}
$$

Since the Wronskian is independent of $x$, we can deduce from the asymptotic form of $\psi_{a \beta}$ that

$$
W\left(\psi_{\alpha+}, \psi_{\alpha-}\right)=2 i k_{\alpha},
$$

and hence that $\psi_{\alpha+}$ and $\psi_{\alpha-}$ are linearly independent except when $k_{\alpha}=0$.

For the potential $q^{0}$, the standard solutions and corresponding reflection and transmission coefficients are

$$
\begin{aligned}
\psi_{\alpha \beta}^{0} & =\exp \left(i \beta k_{\alpha} x\right), \\
r_{+}^{0} & =-\frac{k_{+}-k_{-}}{k_{+}+k_{-}}, \quad t_{+}^{0}=\frac{2 k_{-}}{k_{+}+k_{-}}, \\
r_{-}^{0} & =\frac{k_{+}-k_{-}}{k_{+}+k_{-}}, \quad t_{-}^{0}=\frac{2 k_{+}}{k_{+}+k_{-}} .
\end{aligned}
$$

For the general potential $q$, the coefficients $r_{ \pm}$and $t_{ \pm}$have the following properties.

\section{LEMMA 2.}

(1) $r_{ \pm}$and $t_{ \pm}$are holomorphic functions of $\lambda$ in a strip containing the real axis, cut along $\left(-\infty, q_{+}\right]$, except possibly for poles in the lower half plane. For $\lambda$ in this region the following identities hold:

$$
\begin{gathered}
r_{-}(\lambda) \overline{r_{-}(\bar{\lambda})}+\frac{k_{-}(\lambda)}{k_{+}(\lambda)} t_{-}(\lambda) \overline{t_{-}(\bar{\lambda})}=1, \\
r_{+}(\lambda) \overline{r_{+}(\bar{\lambda})}+\frac{k_{+}(\lambda)}{k_{-}(\lambda)} t_{+}(\lambda) \overline{t_{+}(\bar{\lambda})}=1, \\
t_{+}(\lambda) k_{+}(\lambda)=t_{-}(\lambda) k_{-}(\lambda), \\
k_{+}(\lambda) r_{+}(\lambda) \overline{t_{+}(\bar{\lambda})}=-k_{-}(\lambda) \overline{r_{-}(\bar{\lambda})} t_{-}(\lambda) .
\end{gathered}
$$

(2) $r_{ \pm}$and $t_{ \pm}$are infinitely differentiable on $\left(q_{-}, q_{+}\right)$and

$$
\left|r_{-}(\lambda)\right|=1, \quad q_{-}<\lambda<q_{+} .
$$

(3) If $r_{ \pm}$and $t_{ \pm}$are regarded as functions of $k_{\alpha}$, then they are holomorphic in a neighborhood of $k_{\alpha}=0$. Furthermore,

$$
\lim _{k_{ \pm} \rightarrow 0} r_{ \pm}\left(k_{ \pm}\right)=1, \quad \lim _{k_{ \pm} \rightarrow 0} t_{ \pm}\left(k_{ \pm}\right)=0 .
$$

Proof. When $\lambda$ lies on the cut plane, all the solutions $\psi_{\alpha \beta}$ are holomorphic, so $r_{ \pm}$and $t_{ \pm}$will be holomorphic, except for poles at the zeros of $W\left(\psi_{--}, \psi_{++}\right)$. 
This Wronskian cannot vanish when $\operatorname{Imag} \lambda>0$, for that would imply that $\psi_{--}$ and $\psi_{++}$are linearly dependent, and consequently that the self-adjoint operator $T$ has a complex eigenvalue, because $\psi_{--}$is square integrable at $-\infty$ and $\psi_{++}$at $+\infty$. Hence, any zero of $W\left(\psi_{--}, \psi_{++}\right)$must lie in $\operatorname{Imag} \lambda \leqslant 0$. Nor can zeros lie in $\left(q_{-}, \infty\right)$, for the identities show that $\left|r_{-}\right|$is bounded there.

Both $k_{+}$and $k_{-}$are infinitely differentiable functions of $\lambda$ on $\left(q_{-}, q_{+}\right)$, and $r_{ \pm}$ and $t_{ \pm}$are holomorphic functions of both $k_{+}$and $k_{-}$, regarded momentarily as independent variables. Hence, $r_{ \pm}$and $t_{ \pm}$are infinitely differentiable functions of $\lambda$ on $\left(q_{-}, q_{+}\right)$.

The identities follow trivially from the definitions of $r_{ \pm}$and $t_{ \pm}$and the relations (2.12), (2.13).

Finally, we note that when $k_{\alpha} \rightarrow 0$ the solutions $\psi_{\alpha+}$ and $\psi_{\alpha-}$ become identical, so the limiting values of $r_{ \pm}$and $t_{ \pm}$again follow trivially from the definitions.

\section{The Schrödinger operator and its resolvent kernel}

Consider the symmetric operator on $L^{2}(\mathbf{R})$ defined by

$$
\begin{aligned}
T: K & \rightarrow L^{2}(\mathbf{R}), \\
\phi & \rightarrow\left(-\frac{d^{2}}{d x^{2}}+q\right) \phi,
\end{aligned}
$$

where $K$ is the set of infinitely differentiable functions with compact support. The deficiency indices of $T$ are both equal to zero, so $T$ has a unique self-adjoint extension, its closure, also denoted by $T$. In order to verify this assertion, we need only count the number of solutions of (2.1) which are square-integrable in neighborhoods of $+\infty$ and $-\infty$ when Imag $\lambda \lessgtr 0$. Now

$$
\left|\psi_{\alpha \beta}\right| \sim \exp \left(-\beta \operatorname{Imag} k_{\alpha} x\right) \text { as } \alpha x \rightarrow \infty,
$$

so $\psi_{\alpha \beta}$ is square-integrable near $\alpha \infty$ if and only if $\alpha \beta \operatorname{Imag} \lambda>0$. Thus, in any neighbourhood of $+\infty$ or $-\infty$, only one solution is square-integrable and $T$ does not need to be supplemented by boundary conditions [7, pages 1306 and 1406].

The resolvent of $T$ is a bounded integral operator with kernel [7, page 1329]

$$
R_{\lambda}(x, y)=- \begin{cases}\psi_{--}\left(z_{<}, \lambda\right) \psi_{++}\left(z_{>}, \lambda\right) / W\left(\psi_{--}, \psi_{++}\right), & \operatorname{Imag} \lambda>0, \\ \psi_{-+}\left(z_{<}, \lambda\right) \psi_{+_{-}}\left(z_{>}, \lambda\right) / W\left(\psi_{-+}, \psi_{+-}\right), & \operatorname{Imag} \lambda<0,\end{cases}
$$

where

Note that

$$
z_{<}=\min (x, y), \quad z_{>}=\max (x, y) .
$$

$$
\overline{R_{\bar{\lambda}}^{-}(x, y)}=R_{\lambda}(x, y),
$$

provided that $\lambda$ does not lie on the cut $\left(-\infty, q_{+}\right]$. 


\section{Continuous spectrum of $T$}

The essential spectrum of $T$ coincides with the interval $\left[q_{-}, \infty\right)[7$, pages 1448 , 1438 and 1437].

Let $\Lambda$ denote any open subinterval of $\left[q_{-}, \infty\right)$ and choose

$$
\sigma_{1}(x, \lambda)=\psi_{-+}(x, \lambda), \quad \sigma_{2}(x, \lambda)=\psi_{--}(x, \lambda)
$$

as a basis of solutions of (2.1). After a short manipulation, the resolvent kernel can be written

$$
R_{\lambda}(x, y)=\sum_{i j} \overline{\sigma_{i}(y, \bar{\lambda})} \theta_{i j}^{-}(\lambda) \sigma_{j}(x, \lambda), \quad x<y,
$$

where

$$
\left.\begin{array}{l}
\theta^{-}(\mu-i \nu)=\left[\begin{array}{cc}
\frac{1}{-r_{-}(\mu+i \nu)} & 0
\end{array}\right] / \overline{\left(2 i k_{-}(\mu+i \nu)\right)} \\
\theta^{-}(\mu+i \nu)=\left[\begin{array}{cc}
0 & -r_{-}(\mu+i \nu) \\
0 & 1
\end{array}\right] /\left(2 i k_{-}(\mu+i \nu)\right)
\end{array}\right\}, \quad \nu>0 .
$$

If $\left(\lambda_{1}, \lambda_{2}\right)$ is a subset of $\Lambda$, then the Titchmarsh-Kodaira theorem [7, page 1364] asserts that the spectral measure of $T$ is

$\rho\left(\left(\lambda_{1}, \lambda_{2}\right)\right)=\lim _{\delta \rightarrow 0+} \lim _{\nu \rightarrow 0+} \frac{1}{4 \pi} \int_{\lambda_{1}+\delta}^{\lambda_{2}-\delta} d \mu\left[\begin{array}{cc}1 & -r_{-}(\lambda) \\ \overline{-r_{-}(\lambda)} & 1\end{array}\right]\left[\begin{array}{cc}\overline{k_{-}(\lambda)} & 0 \\ 0 & k_{-}(\lambda)\end{array}\right]^{-1}$,

where

$$
\lambda=\mu+i \nu .
$$

The integrand is continuous on the region

$$
\lambda_{1} \leqslant \mu \leqslant \lambda_{2}, \quad \nu \geqslant 0,
$$

so the limits can be evaluated trivially and

$$
\rho\left(\left(\lambda_{1}, \lambda_{2}\right)\right)=\frac{1}{4 \pi} \int_{\lambda_{1}}^{\lambda_{2}} \frac{1}{k_{-}(\mu)}\left[\begin{array}{cc}
\frac{1}{-r_{-}(\mu)} & -r_{-}(\mu) \\
1
\end{array}\right] d \mu .
$$

This measure is absolutely continuous with respect to Lebesgue measure on $\left[q_{-}, \infty\right)$ and its Radon-Nikodym derivative is

$$
\frac{d \rho}{d \mu}=m(\mu)=\frac{1}{4 \pi k_{-}(\mu)}\left[\begin{array}{cc}
1 & -r_{-}(\mu) \\
\frac{-r_{-}(\mu)}{1} & 1
\end{array}\right] .
$$

If $G$ is a bounded, Borel function with compact support in $\Lambda$, then [7, page 1355] the kernel of $G(T)$ is

$$
G(x, y)=\int_{q_{-}}^{\infty} G(\lambda) e(x, y, \lambda) d \lambda .
$$


where

$$
\begin{aligned}
e(x, y, \lambda) & =\sum_{i j} \overline{\sigma_{i}(y, \lambda)} m_{i j}(\lambda) \sigma_{j}(x, \lambda) \\
& =\operatorname{Real}\left[\psi_{++}(y, \lambda) t_{+}(\lambda) \psi_{--}(x, \lambda)\right] / 2 \pi k_{-}(\lambda) .
\end{aligned}
$$

Since the spectral measure of a set $\{\lambda\}$ containing only one point of $\Lambda$ is clearly zero, because $m$ is continuous at $\lambda$, the operator $T$ cannot have any eigenvalues in $\Lambda[7$, page 1360]. Thus, the essential spectrum of $T$ is purely continuous, except perhaps for the point $\lambda=q_{-}$.

\section{Point spectrum of $T$}

Now let $\Lambda$ denote any open subinterval of $\left(-\infty, q_{-}\right]$. The previous assignment for the solutions $\sigma_{1}(x, \lambda)$ and $\sigma_{2}(x, \lambda)$ is no longer acceptable, because $\psi_{-+}$and $\psi_{--}$are not holomorphic across the branch cut along $\left(-\infty, q_{-}\right.$]. Instead, choose

$$
\sigma_{1}(x, \lambda)= \begin{cases}\psi_{-+}(x, \lambda), & \nu \geqslant 0, \\ \psi_{--}(x, \lambda), & \nu<0,\end{cases}
$$

and

$$
\sigma_{2}(x, \lambda)= \begin{cases}\psi_{--}(x, \lambda), & \nu \geqslant 0, \\ \psi_{-+}(x, \lambda), & \nu<0,\end{cases}
$$

as a basis of solutions of (2.1). These functions are real on $\Lambda$ and have reflection symmetry,

$$
\overline{\sigma_{i}(x, \bar{\lambda})}=\sigma_{i}(x, \lambda)
$$

so are indeed holomorphic in an open region $\Omega$ which contains $\Lambda$. When $\nu>0$, the resolvent kernel can be written

$$
R_{\lambda}(x, y)=\sum_{i j} \overline{\sigma_{i}(y, \bar{\lambda})} \theta_{i j}^{-}(\lambda) \sigma_{j}(x, \lambda), \quad x<y
$$

where

$$
\theta^{-}(\mu+i \nu)=\left[\begin{array}{cc}
0 & 1 \\
0 & -r_{-}(\mu+i \nu)
\end{array}\right] /\left(2 i k_{-}(\mu+i \nu)\right), \quad \nu>0 .
$$

But $\theta^{-}$is real on $\Lambda$ and has reflection symmetry,

$$
\theta^{-}(\mu-i \nu)=\overline{\theta^{-}(\mu+i \nu)}, \quad \nu>0,
$$

a consequence of (4.1) and (6.1), so $\theta^{-}$is also holomorphic in $\Omega$, except for poles of $r_{-}$in $\Lambda$. These isolated points are the eigenvalues of $T$ [7, page 1380]. Since the 
spectrum of $T$ is bounded below [7, page 1459] and the holomorphic function

$$
z(\lambda)= \begin{cases}W\left(\psi_{--}, \psi_{++}\right), & \nu \geqslant 0, \\ W\left(\psi_{-+}, \psi_{+-}\right), & \nu<0,\end{cases}
$$

can only have a finite number of zeros in any bounded region, $T$ has only a finite number of eigenvalues $\lambda_{1}, \lambda_{2}, \ldots, \lambda_{n}$. If

$$
\theta_{t}=\underset{\lambda=\lambda_{t}}{\operatorname{residue}}\left(-r_{-}(\lambda) / 2 i k_{-}(\lambda)\right) \text {, }
$$

then [7, page 1380]

$$
\rho\left(\left\{\lambda_{i}\right\}\right)=\left[\begin{array}{ll}
0 & 0 \\
0 & \theta_{i}
\end{array}\right]
$$

and [7, page 1361]

$$
\chi_{i}(x)=\sqrt{\theta_{i}} \sigma_{2}\left(x, \lambda_{i}\right)
$$

is the normalised eigenvector of $T$ corresponding to the eigenvalue $\lambda_{l}$. Note that all the eigenvalues must be simple.

In analogy with the result for the continuous spectrum, if $G$ is a bounded, Borel function with compact support in $\Lambda$, then the kernel of $G(T)$ is

$$
G(x, y)=\sum_{i=1}^{n} G\left(\lambda_{i}\right) e\left(x, y, \lambda_{i}\right)
$$

where

$$
e\left(x, y, \lambda_{l}\right)=\overline{\chi_{i}(y)} \chi_{\imath}(x)
$$

\section{Nuclearity of $Q$}

To prove the nuclearity of $Q$, we use the following result, proved in [13].

LEMMA 3. Suppose that $Q$ is a bounded integral operator on $L^{2}\left(\mathbf{R}^{n}\right)$ whose kernel, $Q(x, y)$, is twice continuously differentiable with respect to $y$. Construct the kernel

$$
P(x, y)=\left(-\sum_{i=1}^{n} \frac{\partial^{2}}{\partial y_{t}^{2}}+|y|^{p}\right) Q(x, y), \quad p>0 .
$$

If

$$
\int_{\mathbf{R}^{n}} \int_{\mathbf{R}^{n}}|P(x, y)|^{2} d^{n} x d^{n} y<\infty,
$$


so that $P$ is a Hilbert-Schmidt operator, then $Q \in G_{r}$ for all $r>t$, where

$$
t=\frac{2 n(p+2)}{4 p+n(p+2)}
$$

and

$$
s_{k}(Q)=\sigma\left(k^{-1 / t}\right) \text { as } k \rightarrow \infty
$$

Here $s_{k}(Q)$ denotes the $k$ th singular value of $Q$ and $G_{r}$ is the ideal of compact operators whose singular values are $r$-summable.

If we insist that $G$ should be infinitely differentiable, then the integral representation for $Q(x, y)$ is amenable to the standard methods of asymptotic analysis, and a straightforward, but lengthy, calculation leads to the following result.

LemMa 4. Provided $G$ is an infinitely differentiable function,

$$
\left|x^{m} y^{n}\left(\frac{d}{d y}\right)^{2 \prime} Q(x, y)\right| \rightarrow 0, \text { as }|x|,|y| \rightarrow \infty
$$

for all positive integers $l, m, n$.

Hence, for any $p>0$, the kernel $P(x, y)$ satisfies

$$
\left|x^{m} y^{n} P(x, y)\right| \rightarrow 0 \text { as }|x|,|y| \rightarrow \infty,
$$

for all positive $m$ and $n$, and is certainly a Hilbert-Schmidt kernel. In particular, if $p>2 / 3$, then Lemma 3 shows that $Q$ is nuclear. Finally,

$$
\operatorname{trace} Q=\int_{-\infty}^{\infty} Q(x, x) d x
$$

because the kernel of $Q$ is continuous.

\section{Renormalised trace formula}

In the preceding section we assumed that $G$ was infinitely differentiable in order to expedite the asymptotic analysis of $Q(x, y)$ and to establish the nuclearity of $Q$. However, in this section, where we evaluate the integral on the right in (7.1) and show that it indeed reduces to (1.3), we need only require that $G$ be a bounded, Borel function with compact support. Then $G(T)$ is an integral operator whose kernel is

$$
G(x, y)=\sum_{i=1}^{n} \overline{\chi_{i}(y)} G\left(\lambda_{\imath}\right) \chi_{i}(x)+\int_{q_{-}}^{\infty} G(\lambda) e(x, y, \lambda) d \lambda,
$$

so $Q$ is also an integral operator with kernel

$$
Q(x, y)=G(x, y)-G^{0}(x, y) .
$$


We may assume that the support of $G$ lies in one of the sets

$$
\left(-\infty, q_{-}\right],\left[q_{-}, q_{+}\right],\left[q_{+}, \infty\right),
$$

for it is always possible to decompose $G$ into the sum of three functions with this property, so we will prove the theorem for the three cases separately.

(1) Support $G \subset\left(-\infty, q_{-}\right]$.

In this case,

$$
G(x, y)=\sum_{i=1}^{n} \overline{\chi_{i}(y)} G\left(\lambda_{t}\right) \chi_{i}(x)
$$

and

$$
G^{0}(x, y)=0,
$$

because the point spectrum of $T^{0}$ is empty. Hence,

$$
\operatorname{trace} Q=\int_{-\infty}^{\infty} d x \sum_{i=1}^{n} \overline{\chi_{i}(x)} G\left(\lambda_{i}\right) \chi_{i}(x)=\sum_{i=1}^{n} G\left(\lambda_{t}\right),
$$

exactly as expected.

(2) Support $G \subseteq\left[q_{-}, q_{+}\right]$.

The proof in this case is somewhat harder! Write

$$
\operatorname{trace} Q=\lim _{u_{ \pm \rightarrow \pm \infty}} \int_{u_{-}}^{u_{+}} d x \int_{q_{-}}^{q_{+}} d \lambda G(\lambda)\left[e(x, x, \lambda)-e^{0}(x, x, \lambda)\right] .
$$

Since $G$ is integrable and $e$ is continuous for all $(x, \lambda)$ in $\left[u_{-}, u_{+}\right] \times \operatorname{support}(G)$, we may reverse the order of the integrations in (8.1) and write

$$
\operatorname{trace} Q=\lim _{u_{ \pm} \rightarrow \pm \infty} \lim _{\varepsilon \rightarrow 0+} \int_{q_{-}}^{q_{+}} G_{\varepsilon}(\lambda) \int_{u_{-}}^{u_{+}} d x\left[e(x, x, \lambda)-e^{0}(x, x, \lambda)\right],
$$

where

$$
G_{e}(\lambda)= \begin{cases}0, & \lambda<q_{-}+\varepsilon, \\ G(\lambda), & q_{-}+\varepsilon \leqslant \lambda \leqslant q_{+}-\varepsilon, \\ 0, & q_{+}-\varepsilon<\lambda .\end{cases}
$$

This device, which excludes small neighbourhoods of $q_{ \pm}$from the $\lambda$ integration, is necessary because the trick we will use to evaluate the $x$ integration involves differentiation of $r_{ \pm}$and $t_{ \pm}$with respect to $\lambda$, and these functions are not differentiable at $\lambda=q_{ \pm}$. However, we will ultimately be able to evaluate both limits in (8.2).

When $\lambda$ is real, the solutions $\sigma_{i}$ satisfy

$$
\begin{aligned}
& \left(-\frac{d^{2}}{d x^{2}}+q(x)\right) \bar{\sigma}_{i}=\lambda \bar{\sigma}_{i}, \\
& \left(-\frac{d^{2}}{d x^{2}}+q(x)\right) \sigma_{j}=\lambda \sigma_{j} .
\end{aligned}
$$


[16]

Schrödinger operators

153

Differentiate (8.4) with respect to $\lambda$,

$$
\left(-\frac{d^{2}}{d x^{2}}+q(x)\right) \dot{\sigma}_{j}=\lambda \dot{\sigma}_{j}+\sigma_{j} \quad \text { where } \cdot=\frac{d}{d \lambda} .
$$

Multiply (8.3) by $\dot{\sigma}$, and (8.5) by $\bar{\sigma}_{1}$ and subtract the resulting equations to find

$$
\frac{d}{d x}\left\{\dot{\sigma}_{j} \frac{d}{d x} \bar{\sigma}_{\imath}-\bar{\sigma}_{i} \frac{d}{d x} \dot{\sigma}_{j}\right\}=\bar{\sigma}_{i} \sigma_{j}
$$

Hence

$$
\int_{u_{-}}^{u_{+}} e(x, x, \lambda) d x=g\left(u_{+}\right)-g\left(u_{-}\right)
$$

where

$$
g(x)=\sum_{i j} m_{i \jmath}\left[\dot{\sigma}_{j} \frac{d}{d x} \bar{\sigma}_{i}-\bar{\sigma}_{i} \frac{d}{d x} \dot{\sigma}_{j}\right] .
$$

When $\left|u_{ \pm}\right|$is large, we can use the asymptotic forms of $\sigma_{1}$ and $\sigma_{2}$ to evaluate $g\left(u_{ \pm}\right)$. A long and tedious calculation yields the following asymptotic relations, valid when $u_{ \pm} \rightarrow \pm \infty$.

$$
\begin{aligned}
& 2 \pi i g\left(u_{-}\right) \sim \frac{i u_{-}}{k_{-}}+\frac{i}{2 k_{-}^{2}} \operatorname{Imag}\left[r_{-} e^{-2 i k_{-} u_{-}}\right] \\
& 2 \pi i g\left(u_{+}\right) \sim \frac{d}{d \lambda} \log r_{-}-\frac{i}{4} \frac{k_{-}}{\left|k_{+}\right|} e^{-2\left|k_{+}\right| u_{+}}\left|\frac{t_{-}}{k_{+}}\right|^{2}, \quad q_{-}<\lambda<q_{+}, \\
& 2 \pi i g\left(u_{+}\right) \sim \frac{d}{d \lambda} \log \left(t_{-} / i_{-}\right)+\frac{i u_{+}}{k_{+}}-\frac{i}{2 k_{+}^{2}} \operatorname{Imag}\left[r_{+} e^{2 i k_{+} u_{+}}\right], \quad q_{+}<\lambda .
\end{aligned}
$$

We now find that $2 \pi i$ trace $Q$

$$
\begin{aligned}
\lim _{u_{+} \rightarrow \pm \infty} \lim _{\varepsilon \rightarrow 0+} \int_{q_{-}}^{q_{+}} d \lambda G_{e}(\lambda) & {\left[\frac{d}{d \lambda} \log \left(r_{-} / r_{-}^{0}\right)\right.} \\
& -\frac{i k_{-}}{4\left|k_{+}\right|} e^{-2\left|k_{+}\right| u_{+}}\left(\left|\frac{t_{-}}{k_{+}}\right|^{2}-\left|\frac{t_{-}^{0}}{k_{+}}\right|^{2}\right) \\
& \left.-\frac{i}{2 k_{-}^{2}} \operatorname{Imag}\left\{\left(r_{-}-r_{-}^{0}\right) e^{-2 \cdot k_{-} u_{-}}\right\}\right] .
\end{aligned}
$$

https://doi.org/10.1017/S0334270000003647 Published online by Cambridge University Press 
In the second and third terms change the integration variables to $\left|k_{+}\right|$and $k_{-}$ respectively. Thus,

$2 \pi i \operatorname{trace} Q$

$$
\begin{aligned}
=\lim _{u_{ \pm} \rightarrow \pm \infty} \lim _{\varepsilon \rightarrow 0+}\left\{\int_{q_{-}}^{q_{+}} d \lambda G_{\varepsilon}(\lambda) \frac{d}{d \lambda} \log \left(r_{-} / r_{-}^{0}\right)\right. \\
\quad-\frac{i}{2} \int_{0}^{\sqrt{q_{+}-q_{-}}} d\left|k_{+}\right| G_{\varepsilon}\left(q_{+}-\left|k_{+}\right|^{2}\right) \\
\times \sqrt{\left(q_{+}-q_{-}\right)-\left|k_{+}\right|^{2}} e^{-2\left|k_{+}\right| u_{+}}\left\{\left|\frac{t_{-}}{k_{+}}\right|^{2}-\left|\frac{t_{-}^{0}}{k_{+}}\right|^{2}\right\} \\
\\
\left.\quad-i \int_{0}^{\sqrt{q_{+}-q_{-}}} d k_{-} G_{\varepsilon}\left(k_{-}^{2}-q_{-}\right) \operatorname{Imag}\left\{e^{-2 \imath k_{-} u_{-}}\left(\frac{r_{-}-r_{-}^{0}}{k_{-}}\right)\right\}\right\} .
\end{aligned}
$$

Now, $r_{ \pm}$and $t_{ \pm}$are holomorphic functions of $k_{ \pm}$in a neighborhood of $k_{ \pm}=0$, and

$$
\begin{aligned}
& t_{\mp}=k_{ \pm}\left(a+b k_{ \pm}+\cdots\right), \\
& r_{ \pm}=1+c k_{ \pm}+\cdots,
\end{aligned}
$$

so we may allow $\varepsilon \rightarrow 0$ with impunity. The Riemann-Lebesgue lemma shows that the integral containing the oscillatory factor $e^{-21 k_{-} u_{-}}$converges to zero as $u_{-} \rightarrow-\infty$. When $u_{+} \rightarrow+\infty$, the integral containing the factor $e^{-2\left|k_{+}\right| u_{+}}$clearly converges to zero. Hence,

$$
\operatorname{trace} Q=\frac{1}{2 \pi i} \int_{q_{-}}^{q_{+}} d \lambda G(\lambda) \frac{d}{d \lambda} \log \left(r_{-} / r_{-}^{0}\right)
$$

(3) Support $G \subset\left[q_{+}, \infty\right)$.

The proof for this case is almost identical to the last. Let

$$
G_{\varepsilon}(\lambda)= \begin{cases}0, & \lambda<q_{+}+\varepsilon, \\ G(\lambda), & q_{+}+\varepsilon \leqslant \lambda .\end{cases}
$$


Then

$2 \pi i$ trace $Q$

$$
\begin{aligned}
=\lim _{u_{ \pm} \rightarrow \pm \infty} \lim _{\varepsilon \rightarrow 0+} \int_{q_{+}}^{\infty} d \lambda G_{\varepsilon}(\lambda)\{ & \frac{d}{d \lambda} \log \left(\frac{t_{-}}{\overline{t_{-}}} \frac{\overline{t_{-}^{0}}}{t_{-}^{0}}\right) \\
& -\frac{i}{2 k_{+}^{2}} \operatorname{Imag}\left\{\left(r_{+}-r_{+}^{0}\right) e^{2 i k_{+} u_{+}}\right\} \\
& \left.-\frac{i}{2 k_{-}^{2}} \operatorname{Imag}\left\{\left(r_{-}-r_{-}^{0}\right) e^{-2 i k_{-} u_{-}}\right\}\right\} .
\end{aligned}
$$

Note that $t_{-}^{0}$ is real and change the integration variables to obtain

$2 \pi i$ trace $Q$

$$
\begin{aligned}
=\lim _{u_{ \pm} \rightarrow \pm \infty} \lim _{\varepsilon \rightarrow 0+}\left\{\int_{q_{+}}^{\infty} d \lambda G_{e}(\lambda) \frac{d}{d \lambda} \log \left(t_{-} / \overline{t_{-}}\right)\right. \\
-i \int_{0}^{\infty} d k_{+} G_{\varepsilon}\left(k_{+}^{2}+q_{+}\right) \operatorname{Imag}\left\{\left(\frac{r_{+}-r_{+}^{0}}{k_{+}}\right) e^{2 \imath k_{+} u_{+}}\right\} \\
\left.-i \int_{\sqrt{q_{+}-q_{-}}}^{\infty} d k_{-} G_{\varepsilon}\left(k_{-}^{2}+q_{-}\right) \operatorname{Imag}\left\{\left(\frac{r_{-}-r_{-}^{0}}{k_{-}}\right) e^{-2 i k_{-} u_{-}}\right\}\right\} .
\end{aligned}
$$

All the integrals are well defined in the limit as $\varepsilon \rightarrow 0$ and the Riemann-Lebesgue lemma shows the last two terms converge to zero as $u_{ \pm} \rightarrow \pm \infty$. Hence,

$$
\text { trace } Q=\frac{1}{2 \pi i} \int_{q_{+}}^{\infty} d \lambda G(\lambda) \frac{d}{d \lambda} \log \left(t_{-} / \overline{t_{-}}\right) \text {. }
$$

The proof is now complete.

\section{Asymptotic developments of $r_{ \pm}$and $t_{ \pm}$}

In the final section we want to record asymptotic developments for $r_{ \pm}(\lambda)$ and $t_{ \pm}(\lambda)$, valid when $\lambda$ is large and positive. For the analysis the condition on the potential may be weakened and we will only require that

$$
\int_{0}^{\infty}\left|q( \pm(x-a))-q_{ \pm}\right| d x<\infty \text { for all } a \geqslant 0 .
$$


Let us return to the solution of the integral equation (2.2) by iteration. If instead of the estimate (2.6) we use simply

$$
\left|k^{-1} \sin k(x-y)\right| \leqslant|k|^{-1}, \quad k \neq 0,
$$

then it is easy to prove by induction that

$$
\left|k^{n} \psi^{n}(x)\right| \leqslant u(x)^{n} / n !
$$

where now

$$
u(x)=\int_{x}^{\infty}|v(y)| d y
$$

The series (2.5) converges absolutely and uniformly on compact subsets of $x \geqslant 0$, $k>0$. Furthermore, (9.1) shows that the sequence $\left\{\psi^{n}\right\}$ is an asymptotic sequence, and also that the series (2.5) provides the corresponding asymptotic development of $\psi$. However, a more convenient asymptotic development of $\psi$ in descending powers of $k$ can be obtained as follows.

LeMma 5. Suppose that $v$ is infinitely differentiable on $[0, \infty)$, and let

$$
\psi^{n}(x, k)=\exp (i k x)(-2 i k)^{-n} \chi^{n}(x, k) .
$$

Then

$$
\chi^{n}(x, k) \sim \sum_{j=0}^{\infty} \chi^{n j}(x) k^{-j}
$$

where

$$
\chi^{o j}= \begin{cases}1, & j=0 \\ 0, & j>0\end{cases}
$$

and

$$
\chi^{n+1 J}(x)=-\sum_{m=0}^{J}(i / 2)^{m}\left(\frac{d}{d x}\right)^{m-1}\left[v(x) \chi^{n J-m}(x)\right]
$$

Proof. The proof is by induction on $n$. The recursion formula for $\chi^{n}$ is

$$
x^{n+1}(x, k)=\int_{x}^{\infty}(1-\exp [-2 i k(x-y)]) v(y) \chi^{n}(y, k) d y
$$


so, if we assume the form (9.3) for $\chi^{n}$, then

$$
\begin{aligned}
& \chi^{n+1}(x, k) \\
& \quad \sim \sum_{j=0}^{\infty} k^{-j}\left\{\int_{x}^{\infty} v(y) \chi^{n j}(y) d y-\sum_{m=0}^{\infty}(-2 i k)^{-m-1}\left(\frac{d}{d x}\right)^{m}\left[v(x) \chi^{n_{J}}(x)\right]\right\} \\
& \sim-\sum_{j=0}^{\infty} k^{-j} \sum_{m=0}^{\infty}(-2 i k)^{-m}\left(\frac{d}{d x}\right)^{m-1}\left[v(x) \chi^{n_{J}}(x)\right]
\end{aligned}
$$

from which the result follows.

Note the convention used in the lemma, that

$$
\left(\frac{d}{d x}\right)^{-1} f(x)= \begin{cases}-\int_{x}^{\infty} f(y) d y, & \text { if } \operatorname{support}(f)=[-a, \infty), \\ +\int_{-\infty}^{x} f(y) d y, & \text { if } \operatorname{support}(f)=(-\infty,+a]\end{cases}
$$

There is a corresponding development for $\psi_{\alpha \beta}$, the solution of (2.8). We set

$$
v(x)=q(x-a)-q_{+}, \quad x \geqslant 0,
$$

and

$$
k=k_{+}
$$

in the preceding analysis, construct $\chi^{n J}$ from the corresponding solution $\psi\left(x, k_{+}\right)$ of (2.2), and define

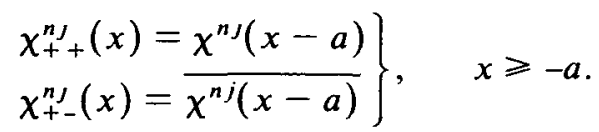

Secondly, we let

$$
v(x)=q(-x+a)-q_{-}, \quad x \geqslant 0,
$$

and

$$
k=k_{-},
$$

construct $\chi^{n \jmath}$ from the corresponding solution $\psi\left(x, k_{-}\right)$of (2.2), and define

$$
\left.\begin{array}{l}
\chi_{-+}^{n \jmath}(x)=\overline{\chi^{n j}(-x+a)} \\
\chi_{--}^{n j}(x)=\chi^{n j}(-x+a)
\end{array}\right\}, \quad x \leqslant a .
$$

We then obtain the following corollary to Lemma 5.

Corollary. Let

$$
\psi_{\alpha \beta}^{n}\left(x, k_{\alpha}\right)=\exp \left(i \beta k_{\alpha} x\right)\left(-2 i \alpha \beta k_{\alpha}\right)^{-n} \chi_{\alpha \beta}^{n}\left(x, k_{\alpha}\right)
$$


Then $\chi_{\alpha \beta}^{n}$ has the asymptotic expansion

$$
\chi_{\alpha \beta}^{n}\left(x, k_{\alpha}\right) \sim \sum_{j=0}^{\infty} k_{\alpha}^{-j} \chi_{\alpha \beta}^{n j}(x) \text { as } k_{\alpha} \rightarrow \infty
$$

where

$$
\chi_{\alpha \beta}^{o j}= \begin{cases}1, & j=0 \\ 0, & j>0\end{cases}
$$

and

$$
\chi_{\alpha \beta}^{n+1 j}(x)=-\alpha \sum_{m=0}^{j}(\beta i / 2)^{m}\left(\frac{d}{d x}\right)^{m-1}\left[\left(q-q_{\alpha}\right)(x) \chi_{\alpha \beta}^{n j-m}(x)\right], \quad \alpha x \geqslant a .
$$

By coupling this asymptotic expansion for $\psi_{\alpha \beta}$ with the expansion of $k_{\alpha}$ in descending powers of $\lambda^{1 / 2}$, we can obtain a similar expansion for $\psi_{\alpha \beta}$ to any order we require. Furthermore, when $|x| \leqslant a$, the expansions for $\psi_{+-}$and $\psi_{-+}$are both valid, so we can estimate $W\left(\psi_{-+}, \psi_{+_{-}}\right)$asymptotically, from which we can obtain developments of $r_{ \pm}$and $t_{ \pm}$. For example, a straight forward but tedious calculation yields

$$
W\left(\psi_{-+}, \psi_{+-}\right) \sim 2 i \lambda^{1 / 2}+\int_{-\infty}^{\infty}\left(q-q^{0}\right)(x) d x+O\left(\lambda^{-1 / 2}\right),
$$

from which it follows that

$$
t_{ \pm}(\lambda) \sim 1+\frac{1}{2 i \lambda^{1 / 2}} \int_{-\infty}^{\infty}\left(q-q^{0}\right)(x) d x+O\left(\lambda^{-1}\right) .
$$

\section{References}

[1] P. Alsholm and T. Kato, "Scattering with long range potentials", Symp. Pure Math. 23 (1973), 393-399.

[2] V. Bargmann, "On the connection between phase shifts and scattering potential", Rev. Modern Phys. 21 (1949), 488-493.

[3] V. S. Buslaev, "Spectral identities and the trace formula in the Friedrichs model", Topics $i n$ Math. Phys. 4 (1971), 43-54.

[4] V. S. Buslaev and L. D. Faddeev, "On trace formulas for Sturm-Liouville differential operators", Dokl. Akad. Nauk SSSR 132 (1960), 13-16.

[5] E. B. Davies and B. Simon, "Scattering theory for systems with different spatial asymptotics on the left and right", Comm. Math. Phys. 63 (1978), 277-301.

[6] V. de Alfaro and T. Regge, Potential scattering (North-Holland, Amsterdam, 1965).

[7] N. Dunford and J. T. Schwartz, Linear operators. Part II (Wiley, New York, 1963). 
[8] L. D. Faddeev, "The inverse problem in the quantum theory of scattering", J. Math. Phys. 4 (1963), 72-104.

[9] L. D. Faddeev and V. E. Korepin, "Quantum theory of solitons", Phys. Rep. 42C (1978), 1-87.

[10] R. Jost, "Über die falschen Nullstellen der Eigenwerte der S-Matrix", Helv. Phys. Acta 20 (1947), 256-266.

[11] M. A. Lohe, "Soliton structures in $P(\phi)_{2}$ ", Phys. Rev. D20 (1979), 3120-3130.

[12] M. A. Lohe and D. M. O'Brien, "Soliton mass corrections and explicit models in two dimensions", Phys. Rev. D23 (1981), 1771-1780.

[13] D. M. O'Brien, "A simple test for nuclearity of integral operators on $L_{2}\left(R^{n}\right)$ ", J. Austral. Math. Soc. A (in press).

[14] S. N. M. Ruijsenaars and P. J. M. Bongaarts, 'Scattering theory for one-dimensional step potentials', Ann. Inst. H. Poincaré Sect. A 26 (1977), 1-17. 